\title{
The elimination of tuberculosis in India
}

\author{
Rajesh Ballal ${ }^{1}$ \\ ${ }^{1}$ Co-Editor, Panacea Journal of Medical Sciences, NKP Salve Institute of Medical Sciences, Nagpur, Maharashtra, India
}

*Corresponding Author:

Email: rajeshballa157@yahoo.com

In March 2017 the Government of India (GoI) announced that the new aim with regard to TB in India was the elimination of TB by 2025. Taking into consideration the various aspects of epidemiology of Tuberculosis, this will require a tremendous effort by all concerned. Tuberculosis is an ancient disease and there is plenty of evidence to support this statement. Most of the understanding about this disease developed during the eighteenth through twentieth century. Workers in this field are too well known to need a mention here. The disease was rampant at one time in the western world and most of these discoveries took place in Europe and USA. These countries also developed a robust notification system which helped to understand the pattern of disease.

The most important finding from these notifications was that Tuberculosis is a disease which comes in epidemics. The epidemic lasts for at least 300 years. This epidemic has been very well described by E R N Griggs in what he coined as the Secular Curve. When Tubercle bacilli are introduced in a virgin population, the infection rapidly spreads to all those exposed. The susceptible develop disease and spread it to others. Over a period of time, without treatment, the susceptible die, the less susceptible develop chronic disease and a few get cured spontaneously. The infectivity increases due to the chronic patients. The mortality is therefore the first to peak and then the morbidity. Gradually as the susceptible die and the herd immunity increases, the disease declines and after a certain point remains endemic with majority of the population infected but immunologically protected, till the next epidemic.

The epidemic started in England in sixteenth century and other the European countries sometime in the seventeenth century and had already crossed the peak and was on the decline, when living conditions improved, understanding of the disease increased and diagnostic tools and chemotherapy were introduced. These further accelerated this decline so that by the middle of twentieth century Tuberculosis ceased to be a major problem in the western world.

In Asia and parts of Africa the epidemic is believed to have started sometime at the end of the nineteenth century and we had yet to reach a peak when chemotherapy was introduced. The poor living conditions and nutrition as well as illiteracy about the disease compounded the problems, leading to the present situation where we harbour a quarter of all Tuberculosis patients in the world. Irregular and incomplete chemotherapy has only worsened the problem. Moreover, since chemotherapy was introduced when the epidemic was on the rise, we had no improvement in herd immunity and are today harbouring a mainly susceptible population.

The current situation in India is an endemicity of epidemic proportions. The National Tuberculosis programme following the National Sample survey was assessed in the early eighties under the three heads- Case finding, Case Holding and Treatment efficacy. It was found that Case finding was 30\%, Case holding 35\% and Treatment efficacy $85 \%$ (in Trial conditions). This meant that out of a potential 100 cases, only 30 were getting diagnosed with the Governmental agencies. Of these 30 only 10 were completing the treatment regularly and for the total duration. Of these 10 only 8 were getting cured. So, practically only $8 \%$ cases were getting effectively cured. Improvement in all three parameters was necessary to create an impact on TB control.

Under the RNTCP introduced at the end of twentieth century, DOTS was introduced and these parameters improved. Encouraged by these results the Government introduced the National Strategic Plan for 2012-17 for a TB Free India through achieving Universal access. The target was to reach $90 \%$ Case finding and to 85 to $90 \%$ Case holding. However despite the best efforts these parameters remain at approximately $60 \%, 80 \%$ and $85 \%$ (in field conditions) respectively. The increase in the effectively treated cases has thus increased from $8 \%$ to about $40 \%$. This has made an impact to the extent that the mortality during last 15 years has almost halved and the incidence is showing an annual decline of $1.2 \%$.

One of the reasons for failing to achieve the target has been the less than desired participation of the Private practitioners. In the new National Strategic Plan (NSP) for 2017-25 therefore the emphasis is going to be on reaching patients seeking care from private providers. The targets for the New NSP include bringing down the Incidence from current 217/lakh population/ year to 77 by 2025 and Prevalence from 320 to 65 (per lakh population); the mortality to be reduced from 32 to 3 (per lakh population).

Nagpur has been chosen for a pilot project for TB free India and the targets are even more ambitious. The target is to bring down the incidence of new cases to $10 \%$ of the present i.e. to 20 per lakh population per year by 2025. A dramatic improvement in all three parameters to close to $100 \%$ would be the basic requirement. But even this may not be enough. We also need to consider the 
large pool of infected individuals who are potential future cases. It is believed that in India almost $100 \%$ population above 40 years of age is infected. Unless tools and tests are developed to identify and treat the infected, who are at risk of developing disease, the target may be difficult. Moreover, an effective vaccine would have to be developed in a hurry to replace BCG which, despite its benefits, fails to prevent adult forms of disease. And to further complicate matters is the man-made problem of Drug Resistance. 